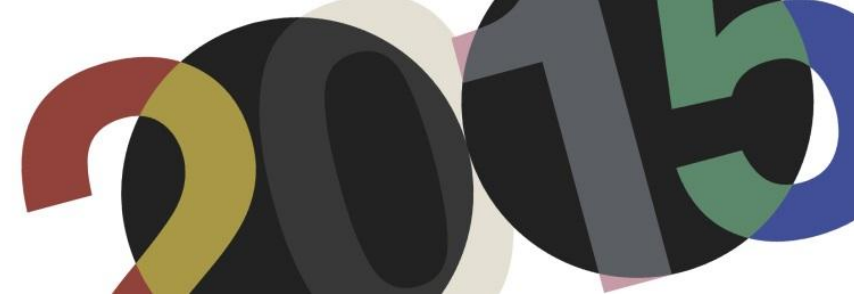

DOI: http://dx.doi.org/10.4995/LC2015.2015.285

\title{
Le Corbusier et les relations avec le Brésil ${ }^{1}$
}

\author{
S.R. Chiarelli, R. Verde Zein
}

Programme d'études supérieures de la Faculté d'Architecture et Urbanisme de l'Université Presbytérienne Mackenzie, Sao Paulo, Brésil

\begin{abstract}
Résumé: Les relations preofessionelles établies par l'architecte suisse-français Le Corbusier avec le Brésil ont été énormes et variées. Ayant été initiées dans les années 1920, elles ont été étendues jusqu'à sa mort en 1965, lorsque son projet de l'Ambassade de la France à Brasilia était en train de se développer (1964). Les informations disponibles sur l'ensemble des projets, des avres et des événements qui montrent la relation de Le Corbusier avec le Brésil ont été dispersés par plusieurs sources, et ils sont étudiés par plusieurs rechercheurs. Cet étude recueille et présente, de façon organisée et systématique, les informations clés sur les événements, en cherchant à élucider les cas où les informations soient différentes ou contradictoires, en présentant un résumé de ses informations sous une forme graphique compacte. L'article propose également une premiàre approche de l'étude d'un cas, celui du projet original de Le Corbusier pour l'Ambassade de la France au Brésil. Une des dernières æevres de l'architecte sera étudiée à la lumière de ses relations antérieures avec le Brésil, en tenant compte de sa position face aux relations stratégiques projectives proposées par l'architecte tout au long de son ouvre, et en considérant d'une façon spécifique mais pas exclusive, les projets realisés ou non, qui ont été élaborés par l'architecte au cours de sa dernière période créative dans les années 1960.
\end{abstract}

Abstract: The preofessionelles relationships established by Swiss-French architect Le Corbusier with Brazil was huge and varied. Having been initiated in the 1920s, they have been extended until his death in 1965, when the project of the Embassy of France in Brasilia was being developed (1964). The information available on all projects, works provided and events that show the relationship Le Corbusier with Brazil were dispersed by several sources, and they are studied by several rechercheurs. This study collects and presents an organized and systematic way, key information about events, seeking to clarify the cases where the information is different or contradictory, presenting a summary of information in a compact graphical form. The article also offers a premiere approach to the study of a case, that the original project by Le Corbusier for the Embassy of France in Brazil. One of the last works provided the architect will be studied in the light of its previous relationship with Brazil, taking into account its position to projective strategic relationship proposed by the architect throughout his work, and considering a specifically but not exclusively, realized or unrealized projects, which were developed by the architect during his last creative period in the 1960s.

Mots clés: Architecture moderne; Brésil; Ambassade de la France; Le Corbusier; cuvres; projets. Keywords: modern architecture; Brazil; Embassy of France; Le Corbusier; buildings; projects.

\section{Introduction}

L'architecte suisse-français Le Corbusier a établi des relations personnelles et professionnelles avec le Brésil depuis les années 1920, et le dernier travail qui l'a été commandé c'était le projet de l'ambassade de la France à Brasilia, la nouvelle capitale du pays. Plusieurs informations sur ces relations entre l'architecte suisse-français et

\footnotetext{
${ }^{1}$ Cet article fait partie de la recherche de doctorat en développement de Mlle Silvia Raquel Chiarelli, sous orientation de la Professeur Docteur Ruth Verde Zein, du programme d'études supérieuresde la Faculté d'Architecture et Urbanisme de l'Université Presbytérienne Mackenzie, et avec l'appui de la Fondation d'Aide à la Recherche de l'état de São Paulo (FAPESP).
} 
le Brésil ont été enregistrées dans les 8 volumes de L'œeuvre Complète ${ }^{2}$ organisés par Le Corbusier, dans des lettres et des photographies disponibles sur le site officiel de la Fondation Le Corbusier ${ }^{3}$; dans les dessins et des notes prises dans ses cahiers de voyage ${ }^{4}$ pendant ses séjours en Amérique du Sud; et aussi dans les textes écrits et publiés par lui-même après une série de dix conférences à Buenos Aires, en Argentine, et à São Paulo et à Rio de Janeiro, au Brésil en 1929, lors de sa première visite à ce «nouveau territoire ». Voici les oeuvres: le livre Précisions: sur un état présent de l'architecture et de l'urbanisme ${ }^{5}$, dans lequel l'auteur raconte ses expériences dans le «nouveau territoire », ses discussions et les dessins qu'il a élaborés au cours de ses conférences; et le texte L'Esprit Sud Américain ${ }^{6}$, qu'il a écrit dans le bateau lors de son retour en France après son premier voyage à l'Amérique du Sud.

En plus des sources de l'architecte, plusieurs chercheurs ont mené des études importantes, globales ou spécifiques, sur ses relations. On peut mentionner le premier livre, Le Corbusier e o Brasil, écrit par les auteurs SANTOS, PEREIRA, PEREIRA et SILVA ${ }^{7}$, qui analyse une grande partie des relations de l'architecte avec le Brésil, en apportant un ensemble détaillé d'informations où sont inclus des dessins et des photographies et des dessins élaborés par l'architecte, et des correspondances échangées entre l'architecte et les brésiliens (Paulo Prado, Lúcio Costa, Oscar Niemeyer, d'entre autres), et entre l'architecte et des étrangers (Fernand Léger, Blaise Cendrars, d'entre autres), qui comme lui avaient aussi des liens avec le pays sud américain et, à cause de ça, ont enregistré des dialogues sur ce «nouveau territoire».

La première publication d'un autre auteur à propos de ce sujet est probablement le livre Leitura Crítica de Le Corbusier, écrit par Pietro Maria Bardi, sous forme compacte, publé en 1950, à l'occasion de l'exposition itinérante de l'architecte (y inclus les peintures, les sculptures et les oeuvres d'architecture). L'exposition a eu lieu du 5 juillet 1950 au 5 mai de l'année suivante, environ deux ans après l'inauguration du Musée d'Art de São Paulo - MASP en 1948, où se trouvait à l'époque son premier siège, à la rue 7 de Abril, ayant pour titre « Nouveau Monde dans l'espace de Le Corbusier ». Bardi était le directeur du Musée d'Art de São Paulo et il connaissait déjà l'architecte suisse-français: les correspondances entre eux ont été publés plus tard dans le livre Lembranças de Le Corbusier: Atenas, Itália, Brasil. ${ }^{8}$

Les relations de Le Corbusier avec le Brésil sont aussi thème de l'interview que l'architecte Ítalo Campofiorito a donné à Hugo Segawa, quand Campofiorito ${ }^{9}$ raconte ses souvenirs de la visite de Le Corbusier à Brasilia et à Rio de Janeiro en 1962, qu'il a accompagné de très près, à la demande de Lucio Costa et Oscar Niemeyer.

\footnotetext{
2 JEANNERET-GRIS, Charles-Édouard. Le Corbusier, œuvre complète 1910-1965. Zurich. Artemis, 1967, 8 volumes.

${ }^{3}$ Cf. www.fondationlecorbusier.fr, Accédé le 21 mai 2015.

${ }^{4}$ Le Corbusier Carnets. New York / Londres: The Architectural History Foundation / Dessin et Tolra, 1981-1982, 4 volumes, I. 1914-1948; 2. 1950-1954; 3. 1957-1964.

${ }^{5}$ LE CORBUSIER. Precisões : sobre um estado presente da arquitetura e urbanismo. Traduction: Carlos Eugênio Marcondes de Moura. São Paulo : Cosac Naify, 2004. (Éd. Orig. : Précisions : sur un état présent de l'architecture et de l'urbanisme, 1930).

${ }^{6}$ In : SANTOS, Cecília Rodrigues dos ; PEREIRA, Margareth Campos da Silva ; PEREIRA, Romão Veriano da Silva ; SILVA, Vasco Caldeira. Le Corbusier e o Brasil. São Paulo : Tessels, 1987, p. 68-71.

${ }^{7}$ SANTOS, Cecilia Rodrigues dos ; PEREIRA, Margareth Campos da Silva ; PEREIRA, Romão Veriano da Silva ; SILVA, Vasco Caldeira. Le Corbusier e o Brasil. São Paulo, Tessela, 1987.

${ }^{8}$ Livre et des documents correspondants consultés dans la collection du Centre de Photographies et de la Bibliothèque et Centre de Documentation du Musée d'Art de São Paulo.

${ }^{9}$ Interview de Italo Campofiorito à Hugo Segawa. A viagem de Le Corbusier ao Brasil em 1962. In : revista Projeto, une édition spéciale pour commémorer les 100 ans de Le Corbusier, São Paulo, n. 102, 1987, p. 113-116.
} 
D'entre les informations et enregistrements sur les relations entre l'architecte et l'Amérique du Sud ${ }^{10}$, dont le traitement est toujours en cours, on peut y inclure le livre Le Corbusier y Sudamérica: viajes y proyectos ${ }^{l 1}$ dans lequel les voyages de Le Corbusier vers le territoire sud américain sont mentionnés, y compris les trois fois qu'il a visité le Brésil, en 1929, 1936 et 1962.

Marcelo Puppi a publié un résumé de la vaste recherche ${ }^{12}$ qu'il a récemment terminé, dans laquelle il précise certaines informations importantes sur les relations entre le projet de Lúcio Costa et le projet de Le Corbusier et son studio pour la Maison du Brésil, dans la Cité Universitaire International de Paris (France) dans les années 1953-1956.

Pour le présent travail sont de majeure importance les études sur Le Corbusier qui sont en train d'être développées par l'architecte et professeur colombien à la Pontificia Universidad Católica de Chile (PUC-Chile) et qui sont faites par Ricardo Daza dans sa thèse de doctorat et aussi dans une recherche récente sur le voyage de l'architecte suisse-français à l'Est ${ }^{13}$. Ça a été d'un grand intérêt la façon dont cet auteur a travaillé les relations croisées entre les plusieurs projets et les dessins de Le Corbusier, lesquelles seront la base de cette proposition de recherche.

Cette étude a également éxaminé les informations sur d'autres recherches en cours, dont nous avons déjà pris la connaissance ${ }^{14}$. Par exemple, le travail de l'architecte chilien et professeur de la Pontificia Universidad Católica de Chile (PUC-Chile), Dr. Francisco Chateau, qui étudie aussi le projet proposé par Le Corbusier pour l'Ambassade de France à Brasilia, et celui qui, en effet, a été réalisé, proposé par De La Fuente, mais avec une approche différente proposée par cette recherche ${ }^{15}$. Nous pouvons aussi faire référence aux recherches en cours dans la thèse de doctorat qui est menée au Brésil par l'architecte colombienne Ingrid Quintana Guerrero, sur les relations et les échanges entre Le Corbusier et ses collaborateurs de l'Amérique Latine qui ont eu lieu dans son bureau situé dans la Rue de Sèvres, à Paris ${ }^{16}$. Le cadre sera aussi révisé avec les données obtenues de la recherche de sources primaires, qui sont disponibles à partir de plusieurs sources, dont la principale c'est la collection de la Fondation Le Corbusier à Paris, en France; outre les archives de l'actuelle Ambassade de la France au Brési ${ }^{17}$ et les archives du Ministère des Affaires Étrangères, les deux situés à Brasilia, qui permettront baser et complémenter les interpretations et les analyses proposées par cette recherche en cours.

A partir des références bibliographiques déjà consultées, une liste des oeuvres et des événements qui lient l'architecte au Brésil et qui, jusqu'à ce moment-là étaient dispersés dans plusieurs sources différentes, a été organisée de la manière la plus complète et systématique possible. Cette recherche préliminaire a constaté que,

\footnotetext{
${ }^{10}$ Les références mentionnées ci-dessus ont été soigneusement recherchées ; les prochaines références sont encore entrain d'être consultées et recherchées.

${ }^{11}$ OYARZÚN, Fernando Pérez, Le Corbusier y Sudamérica : viajes y proyectos. Santiago de Chili : Ediciones ARQ. 1991.

12 PUPPI, Marcelo. Espaços Inacabados: Le Corbusier, Lúcio Costa e a saga da Casa do Brasil, 1953-1956. Revue ARQTEXTO (PROPAR/UFRGS), Porto Alegre, n. 12, 2008, p. 160-203.

${ }^{13}$ Ricardo Daza est co-directeur de cette recherche et l'auteur de la thèse El viaje de Oriente. Charles-Édouard Jeanneret (Le Corbusier) y Auguste Klipstein, gagnante du concours de meilleure thèse d'Espagne 2011.

${ }^{14}$ Les contacts personnels avec ces auteurs ont été progressivement mis en place par les auteurs, qui prévoient des nouvelles visites sur le terrain au Chili.

${ }^{15}$ Francisco Chateau est professeur à Pontificia Universidad Católica de Chile, et il a écrit l'article « Yellow Peripherical Distinction. Guillermo Jullian en el encuentro del Team X en Berlim». 1973, dans la revue Masillia 2007.

${ }^{16}$ Recherche de doctorat en cours à la Faculté d'Architecture et Urbanisme de l'Université de São Paulo (FAU-USP), sous l'orientation du professeur Dr. Paulo Bruna.

${ }^{17}$ Projetée, après le décès de Le Corbusier, par l'architecte chilien, et par son collaborateur dans son atelier Rue de Sèvres, Guillermo Jullien De La Fuente.
} 
bien que certaines de ses informations soient répétées et corroborent en deux ou plusieurs références, l'ensemble ne se produit pas en tant que telle dans toutes les sources. On a également constaté l'existence de quelques informations différentes, parfois contradictories, d'autres fois complémentaires, selon différentes sources. Finalement, on a noté que quelques informations sont perceptibles dans une référence, n'étant pas mentionnées par d'autres, ni même par les archives de la Fondation, et qui donc ont besoin de passer par une vérification ultérieure.

Toutes les informations obtenues juqu'à présent sur les relations personnelles et professionnelles entre Le Corbusier et le Brésil ont été collectées et consolidées. La chronologie des événements et une table ont été ellaborées avec des informations systématiques sur les relations professionneles (visites, projets, ordres, expositions, et etc.). Les deux pourront éventuellement être corrigées et complémentées par la recherche avec la vérification d'autres sources. Une table a été éllaboré par les auteurs avec des informations systématiques sur: a) le type de document (lettre, croquis, dessin, projet, texte, et etc.) où est mentionné le projet ou l'événement qui lie l'architecte suisse-français au Brésil; b) les pages dans lesquelles se trouvent les références de toutes les sources accedées; c) une brève description des informations contenues dans ces fichiers. Elle permet de vérifier brièvement quelles sources mentionnent chaque projet et/ou événement et quel type d'information apparaît dans chacune de ces sources.

La table s'est révélée un outil très utile pour recueillir et consolider les données déjà obtenues, et suggère que les aspects de la recherche doivent être approfondis pour vérifier et confirmer la pleine existence de ces informations. Il y a des données sur lesquelles très peu d'information a été trouvé dans les références et les fichiers consultés jusqu'à ce moment-là; il y a aussi d'autres avec des informations peu précises jusqu'à présent.

Une partie importante des données obtenues est trouvée seulement dans la liste des fichiers de l'architecture de l'index par ordre alphabétique disponible sur le site de la Fondation Le Corbusier ${ }^{18}$. Ces données ont été surlignés en rouge dans la table, et elles seront objet de recherche dans les archives de la Fondation.

Il y a des informations différentes ou divergentes à propos du même événement; ces cas sont surlignés en vert. C'est le cas, par exemple, de l'exposition des oeuvres de Le Corbusier au Musée d'Art de São Paulo - MASP en 1950. Sur la liste des expositions des oeuvres de l'architecte (expositions oeuvres L.C.) disponibles sur le site de la Fondation Le Corbusier on ne trouve que l'information que Le Corbusier aurait fait une exposition de ses peintures à São Paulo en 1950. Cependant, les livres écrits par SANTOS et alii (1987) et BARDI (1984) révèlent plus de détails sur l'exposition. Les deux informent qu'il s'agit de l'exposition « Nouveau Monde de l'Espace» (New World of Space), organisée par The Institute of Contemporary Art de Boston, États-Unis en 1948, et qui avait déjà passée par plusieurs musées disponibles sur le site de la Fondation Le Corbusier on ne trouve que l'information que Le Corbusier aurait fait une exposition de ses peintures à São Paulo en 1950. Cependant, les livres écrits par SANTOS et alii (1987) et BARDI (1984) révèlent plus de détails sur l'exposition.

Les deux informent qu'il s'agit de l'exposition «Nouveau Monde de l'Espace» (New World of Space), organisée par The Institute of Contemporary Art de Boston, États-Unis en 1948, et qui avait déjà passée par plusieurs musées américains et est arrivée au Masp en 1950.

Seulemente Bardi, e directeur du musée à l'époque, mentionne que l'exposition a été ouverte du 5 juillet 1950 au 5 mai de l'année suivante et qu'elle avait, en plus du titre, un sous-titre: «Some Day through Unanimous Effort Unity Will Reign Once More in the Major Arts: City Planning and Architecture, Sculpture, Painting », ce qui indique que l'exposition comprenait des oeuvres de peinture, sculpture et architecture de Le Corbusier. Il

${ }^{18}$ Cf. www.fondationlecorbusier.fr, Archives $\rightarrow$ Architecture $\rightarrow$ Index Alphabétique (recherche menée le 19 avril 2015). 
mentionne également la publication du catalogue «Lecture Critique de Le Corbusier » (1951), écrit par Bardi à l'occasion de l'exposition pour la divulguer. Seulement SANTOS et alii, dans Le Corbusier e o Brasil, ont inclus des annotations sur une liste de noms des tableaux qui étaient dans les boîtes débarqués au port de Santos, et qui apportaient le matériel à être exposé dans le Musée d 'Art de São Paulo. Ces informations, incluses dans les deux références bibliographiques, semblent se complémenter.

Afin de clarifier tous les doutes, quelques documents présents dans la collection du Centre de Photographie, dans la Bibliothèque et le Centre de Documentation du MASP ont été consultés. Les correspondances échangées entre Pietro Maria Bardi et The Institute of Contemporary Art de Boston ont été trouvés, datées des années entre 1948 et 1951, et qui ont enregistré les procedures nécessaires pour que l'exposition vienne au Brésil, en plus de documents fiscaux, des reçus, des tickets, des notes manuscrites écrites par Bardi, qui indiquent les oeuvres de chacune des boîtes livrés au MASP pour l'exposition de Le Corbusier. Les informations enregistrées sont d'accord avec celles publiées dans le livre Le Corbusier e o Brasil. Quelques autres documents consultés également confirment l'importation des oeuvres de l'exposition itinérante de Le Corbusier, envoyées par le musée Nord Américan au musée brésilien. Certaines de ces correspondances ont été sélectionnées pour être publiées dans son intégralité ici (Figures 2 à 4). Elles sont datées du 8 mai 1950, le 10 mai 1950 et le 18 mai 1950.

Les deux références bibliographiques mentionnent que l'architecte a refusé l'invitation de Bardi de venir au Brésil pour voir l'inauguration de sa propre exposition. Et aussi que, dans la lettre envoyée au directeur du musée, il se plaignait des brésiliens. Cependant, cette lettre n'a pas été trouvé parmi la collection du musée ${ }^{19}$. Le Corbusier faisait probablement allusion aux développements concernant ses attitudes envers les projets du Ministère de l'Éducation et de la Santé et de la Cité Universitaire, à Rio de Janeiro, qui étaient l'objet de sa visiteen 1936; et aussi sur l'exposition de l'architecture moderne brésilienne sponsorisée par l'Ambassade du Brésil à Paris.

Selon les auteurs SANTOS et alii :

«il (Le Corbusier) va plus loin quand il propose aussi (à Bardi) l'acquisition de sa toile 'Nature Morte Pleine de Lumière' de la part de MASP, comme une forme de lui compenser financièrement pour les injustices alleguées. L'idée est immédiatement acceptée par le critique italien et le tableau ira ainsi enrichir la collection du nouveau musée, après l'ouverture d'une action pour réunir des fonds nécessaires pour l'acquisition ». (1987: 222)

Cependant, il y a des désaccords sur l'étape finale du processus d'achat et de vente du tableau. Dans le texte cidessus SANTOS et alii (1987) ils impliquent a vendu le tableau pour le musée e que celui-ci est devenu une partie de la collection du musée. Par contre, BARDI (1984) révèle que, malgré les efforts du directeur, du groupe d'architectes et des entreprises prêtes à aider financièrement dans le procès d'acquisition, la toile n'aurait pas été acquise par le musée.

"On a essayé d'acheter une des peintures exposées, mais ça n'a pas été possible puisque la souscription lancé n'a pas réussi, malgré la participation de Rino Levi,

\footnotetext{
${ }^{19}$ Il n'en reste qu'à faire une recherche dans les arqchives personnels de Pietro Bardi, conservés par l'Institut Lina Bo et Pietro Maria Bardi, qui n’ont pas été systématisés que très récemment.
} 
Severo Gomes, Warchavchik, Jacques Pilon, Lina Bardi, Aldo Magnelli, Luis Medici, Henrique Mindlin, Lucjan Korngold et Marcelo Roberto (...)». 1984 :106) ${ }^{20}$

Les informations indiquées ci-dessus ne font référence que dans un cas en particulier. Il s'agit seulement d'une petite partie de ce processus complexe, en cours, de la recherche, vérification et consolidation des informations sur les interactions entre Le Corbusier et le Brésil, consideré comme la base indispensable pour la compréhension et lecture de la dernière de ses oeuvres brésiliennes: le projet, non réalisé, de l'Ambassade de la France à Brasilia.

\section{Le voyage de 1962 : la préparation du projet de l'Ambassade}

En décembre 1962 Le Corbusier visite le Brésil pour la troisième fois; plus précisement les villes de Rio de Janeiro et la nouvelle capitale du pays, inaugurée en 1960, la ville de Brasilia. Sa visite fut accompagnée par Lucio Costa, Oscar Niemeyer et, en spécial, par Italo Campofiorito. Une des raisons principales pour cette visite était de connaître le terrain destiné au projet de l'Ambassade de la France au Brésil. Il s'agit du dernier d'une série de projets et travaux, réalisés et non réalisés, conçue par architecte suisse-français pour le Brésil. Il s'agit également d'un des derniers projets de sa vie. Il a été ellaboré par Le Corbusier entre 1964 et 1965, après son dernier voyage au Brésil, et un peu avant de sa mort, au mois d'août 1965.

Sa visite au terrain, à Brasilia, où le projet de l'Ambassade serait construit, a été enregistrée par des dessins contenus dans un de ses «carnets de voyage », ce qui confirme que Le Corbusier avait l'habitude de les faire pendant tous ses voyages. Lors de cette visite il était accompagné de Italo Campofiorito, à l'époque chef du Service de l’Urbanisme Métropolitain de la ville de Brasilia.

Selon Campofiorito, lors de son interview à Hugo Segawa $(1987)^{21}$, il aurait été choisi par Oscar Niemeyer et Lucio Costa pour accompagner l'architecte étranger pendant son séjour dans notre pays. Dans ce texte, il remémore les moments pendant les visites au musée d'Art Moderne à Rio de Janeiro, conçu et construit par Alfonso Eduardo Reidy selon les doctrines du «maitre » suisse-français, et à la Maison des Canoës », d'Oscar Niemeyer, avec toutes les courbes et la délicatesse inhérentes à son « disciple » brésilien. Il se souvient aussi du moment où il a laissé l'architecte dans la compagnie de Lucio Costa, dans l'immeuble du Ministère de l'Éducation et de la Santé. Il s'agissait de la première fois que Le Corbusier visitait l'édifice après sa construction. Selon Campofiorito, Le Corbusier était fier de connaître cet édifice, qui n'avait pas été projeté exclusivement par lui parce que c'était le travail conjoint d'un group de jeunes architectes brésiliens qui se sentaient comme des «disciples» du «maitre » suisse-français et lesquels avaient, sur le point de vue de son «maître », réunit et construit leurs idées très bein, et bien avant qu'il même le faisait.

Selon Campofiorito, Le Corbusier a exprimé les mêmes sentiments lorsqu'il a connu Brasilia. Il s'agissait d'une ville qui avait été construite dans l'espace de trois ans, et dont la construction était surtout basée sur ses propres idées, datées de 40 ans auparavant, pour la «Ville contemporaine de trois millions d'habitants » (1922). Le Corbusier serait aussi surpris avec la construction de l'Université de Brasilia - UnB - plus spécifiquement avec le Centre de Planification Oscar Niemeyer - CEPLAN, construit en 1962 construit selon le projet d'Oscar Niemeyer, et dans lequel on a utilisé des matériaux pré-fabriqués. Pour Campofiorito, l'architecte a eu un regard

\footnotetext{
${ }^{20}$ Quelques documents existents dans la Bibliothèque et Centre de Documentation du MASP r'vèlent quelques parts du procès de vente de la toile de Le Corbusier (appelée dans la lettre comme "Nature morte pleine d'espace »).

${ }^{21}$ SEGAWA, Hugo. Entrevistas e Testemunhos :A viagem de Le Corbusier ao Brasil em 1962. In : Projet, São Paulo, n. 102, 1987.
} 
d'admiration sur toute la ville et ses immeubles, où il a visualisé la maîtrise des jeunes architectes qui suivaent da doctrine. Selon les mots du propre Campofiorito, Le Corbusier aurait dit que:

«Je ne ferais pas comme ça parce que je suis dur, je suis lourd. Vous êtes marrants, vous les brésiliens. Vous êtes délicats, vous êtes presque féminins. Rodrigo (Melo Franco de Andrade), Lucio (Costa), Oscar (Niemeyer). Et même vous, Italo, vous êtes très délicat. Je suis un brute; ici ; je n'auras pas fait comme ça, je n'aurais pas fait ça de cette manière si délicate, les colonnes d'Oscar sont très délicates, le sol est très délicat. J'aurais fait construire la Place des Trois Pouvoirs avec des grandes dalles de béton et le long de l'asphalte, comme dans les aéroports ». SEGAWA, 1987 :113).

Campofiorito se souvient que, pendant la visite de Le Corbusier à Brasilia, le Ministre de la Culture à l'époque, Darcy Ribeiro aurait « commandé » à Le Corbusier les projets pour l'Ambassade de la France e aussi celui de la Maison de Culture Française; cependant, il n’y a aucun registre postérieur sur ce deuxième projet. Malgré la surprise de cette information (vue que le seul client avec le pouvoir de commander ce type de travail était le gouvernement français), les auteurs SANTOS et alii la mentionne aussi, et ils ajoutent qu'il y a eu également une mention d'un ordre pour les projets du Musée National de Brasilia, ou Centre National de Culture Brésilienne, ou Maison de Culture du Brésil. Selon eux, lors du voyage de 1962 Niemeyer aurait parlé à Le Corbusier sur cette probable commande, et sur le projet pour l'Ambassade de la France. D'après les auteurs, pendant ses derniers deux ans de vie, en Europe, Le Corbusier a été informé que ces trois projets ne seraient pas réalisés.

Quelques décennies plus tard, Pietro Maria Bardi, qui se prenait pour un ami de Le Corbusier, a publié dans son livre Lembranças de Le Corbusier : Atenas, Itália, Brasil, que l'architecte suisse-français avait visité, à Rio de Janeiro, le Ministère de l'Éducation et de la Santé, le Musée d'Art Moderne, l'Ensenble Pedregulho, les maisons de Sérgio Bernardes et celle de Jorge Moreira (1984 : 111 a 115), et qu'il aurait également visité Brasilia ayant l'intention de connaître la nouvelle capitale et le terrain où il allait projeter l'Ambassade de la France ${ }^{22}$.

Dans les livres Le Corbusier e o Brasil (1986) et aussi dans le livre de DI MATTEO et TIDORI (org.), Embaixada da França: Brasilia (2009), les auteurs parlent sur les efforts subis, à partir de 1955, dans le sens de lier son nom à la construction de la nouvelle capitale brésilienne. Les auteurs indiquent quelques parties des procédures de la commande du projet de l'Ambassade de France faite par le Ministère des Affaires Étrangères de la France à l'architecte; et ils relatent quelques difficultés qui ont conduit à prendre la décision finale de ne pas réaliser le projet après la mort soudaine de l'architecte. Ces informations se répètent partiellement dans les deus références bibliographiques cités, et les deux ont inclus d'autres détails qui se complétent mutuellement. La prise de décision de ne pas construire le projet du «maître » est traitée d'une façon plus détaillée dans le livre Embaixada da França: Brasilia, où sont révelés quelques faits qui se sont produits après la mort du «maître », jusqu'en 1970, quand un nouveau projet a été commandé par son «héritier» et bras droit dans la Rue bde Sèvres, l'architecte chilien Guillermo Jullian de La Fuente, et que ce fait se serait passé après la pression exercé par le gouvernement brésilien pour que le gouvernment français commence la construction de son ambassade dans la capitale déjà consolidée. Selon SANTOS et alii, le projet de Le Corbusier n'aurait pas été effectué parce que le gouvernement français «n'a jamais fait des efforts pour la réalisation de ce projet » (1987 :255)

\footnotetext{
${ }^{22}$ Ni Campofiorito ni Bardi avaient l'habitude de mentionner des faits de leur mémoires, une raison qui peut expliquer la différences dentre les informations. Celui-ce, et bien d'autres détails seront également vérifiés tout au long de la recherche.
} 
«Dans la lettre adressée à (André) Malraux (Ministre d'État de la France) juste après son retour du Brésil, l'architecte proteste amèrement à cause du mépris et du manque de reconnaissance de son travail de la part de la France, une complainte justifiée mais, d'une certaine manière, également prémonitoire vue que, après sa mort, même le contrat de construction de l'Ambassade de ce pays à Brasilia, le dernier projet qui, si on prend en considération la persévérance des suisses, pourrait être mis à jour, ce qui aurait couronner les efforts pour laisser une marque concrète du passage de l'architecte par le Brésil. » (1987 :259).

Il est intéressant de noter que le gouvernment suisse aurait commandé à Le Corbusier, en 1961, le projet de l'Ambassade de la Suisse à Brasilia. Un tel projet n'a jamais été éllaboré par le «maître », qui aurait refusé cette proposition en affirmant qu'il avait trop de travail à l'époque. Le projet de 1'Ambassade de la Suisse est mentionné dans le livre de SANTOS etb alii, qui inclut la correspondence de Jacob Ott à Le Corbusier, datée du 20 janvier 1961 (1987 : 289); et fait partie aussi de la liste de projets non réalisés disponible sur le site de la Fondation Le Corbusier.

Pendant son séjour au Brésil, Le Corbusier n'a pas donné des interviews à aucun journal brésilien; cependant, avant de retourner en Europe, il a fait publier à la prsse brésilienne le texte suivant ${ }^{23}$ comme forme de remercier le pays qu'il a toujours consideré un « débiteur »:

«Le Corbusier dit que Brasilia est magnifique et qu'elle lui parle au coeur.

Le Corbusier, le fameux architecte français qui est venu au Brésil pour étudier et recueillir des éléments avec lesquels il veut projeter l'édifice de l'Ambassade de la France à Brasilia, a retourné samedi soir à Paris. Dans son message d'adieu, en s'adressant aux brésiliens, Le Corbusier a souligné: 'Pour ce grand voyageur que je suis, il y a dans le planisphère, entre les montagnes, sur les plaines et plateaux où les grandes rivières courrent vers la mer, des zones privilégiées. Le Brésil est un de ces endroits très chaleureux et généreux, qu'on aime appeler d'ami. Brasilia est construite. J'ai vu une ville déjà née. Elle est magnifique d'invention, de courage, d'optimisme; et elle me parle au coeur. C'est l'oeuvre de mes grands amis et (à travers les ans) de compagnons de lutte, Lucio Costa et Oscar Niemeyer. Dans le monde moderne, Brasilia est unique. A Rio, il y a le Ministère de 1936-45, il y a des oeuvres de Reidy, le monument en honneur aux morts de la guerre. Il ya beaucoup de témoignages. Ma voix est celle d'un voyageur de la terre et de la vie. Mes amis du Brésil : laissez-moi vou dire merci $\gg{ }^{24}$

Dans ce texte, Le Corbusier ne fait aucune critique; au contraire, il remercie et fait des compliments au pays et aux brésiliens, et il se souvient de ses amis, sans créer des controverses. Ce message serait son dernier contact publique avec le pays. A cette époque, encore un de ses projets pour le pays, né également d'une commande idéalisée, mais pas clairement établie, n'aurait pas du succès non plus. A sentir l'optimisme de son ton, peut-être

\footnotetext{
${ }^{23}$ Texte aussi publié dans les livres: Le Corbusier e o Brasil (1986 : 292) et Embaixada da França: Brasilia (2009: 13)

${ }^{24}$ Journal O Globo, le 31 décembre 1962. Disponible dans la collection de la Bibliothèque et Centre de Documentation du MASP.
} 
Le Corbusier croyait alors, en effet, que le panorama de frustrations serait finalement surmonté, et finalement une de ses oeuvres serait construite au Brésil.

Cependant, les termes de cette commande du projet de l'Ambassade de la France pour Le Corbusier continue à être peu défini par les sources consultés, et la responsabilité d'avoir des précisions sur ce thème est à la recherche, en cherchant des documents qui prouvent quels personnages y sont impliqués, et en menant également une investigation sur quelles raisons ont fait que le projet proposé par Le Corbusier n'ait pas été accepté. En outre, la recherche ira vérifier les questions relatives au projet lui-même: le programme, le site, les conditions, et comment éventuellement elles sont présentes dans la commande du client, et/ou ont été conçues par Le Corbusier à partir de ses propres idées. Pour cela, il est probable qu'il sera nécessaire de faire une comparaison avec le projet finalement éllaboré par Guillermo Jullian de La Fuente, construit entre 1972-1974², bien que ce fait ne soit pas l'objectif principal de la présente recherche, mais l'oeuvre Corbusienne.

\section{L’Ambassade de la France et d'autres œuvre}

Jusqu'à ce moment, la recherche a déjà organisé les bases sur lesquelles on a l'intention d'étudier de forme critique le projet pour l'Ambassade de la France, éllaboré par Le Corbusier, et qui englobe la reconnaissance de l'ensemble de son oeuvre, de forme à établir, ou proposer, des relations entre quelques unes de ses oeuvres, distribuées au long de toute sa carrière, et quelques unes de ses oeuvres, réalisées dans la même période, et qui ont été soigneusement étudiées et sélectionnées. On cherche, par conséquent, contribuer à la compréhension des présences constantes dans les solutions formelles, compositives et constructives de son oeuvre, et qu'elles aient de la signification pour la comprehénsion de celui qui est un des derniers projets de Le Corbusier: l'Ambassade de la France au Brésil.

La sélection des oeuvres à être considerées comme «significatif » pour l'étude présente a suivi les critères suivants:

a. Des oeuvres construites et non construites et les événements qui font une relation de l'architecte avec l'Amérique du Sud;

b. Des oeuvres construites et non construites et les événements qui font une relation de l'architecte avec le Brésil;

c. Des oeuvres internationales construites et non construites par l'architecte qui semblent présenter une relation avec le projet pour l'Ambassade de la France, sous le point de vue de la construction et de la composition architecturale.

Les oeuvres internationales en référence dans le dernier critère ont été sélectionnées à partir d'autres critères qui traitent de solutions architecturales (programmatique, constructive, compositive, et etc.) présentes dans le projet pour l'Ambassade de la France:

a. Projets d'ambassades;

b. Programme résidentiel;

c. Relation avec l'échelle urbaine;

d. Premières relations avec le Brésil;

e. Structure «dom-ino»;

${ }^{25}$ DI MATTEO, Colette, TIDORI, Jean-Martin (org.). Embaixada da França. L'Ambassade de la France. Brasilia. São Paulo: Imprensa Oficial do Estado de São Paulo, Instituto Totem Cultural, 2009. 
f. Présence des «brise-soleil »;

g. Présence de volumes cylindriques;

h. Présence de pied-droit double ou triple;

i. Accès par rampes / « promenade architecturale »;

j. Présence de dalles «perforées »/ des cours.

Ayant pour base ces critères, on a organisé un panneau d'oeuvres et d'événements (Figure 5) unis par une ligne du temps qui a, comme point de départ, la naissance de l'architecte et comme final l'année de sa mort.

Ensuite, les événements ont été exclus pour permettre de visualiser seulement les oeuvres qui semblent présenter quelque relation avec le projet pour l'Ambassade de la France, et on a divisé le panneau en suivant la même division par volumes présente dans L'Oeuvre Complète de Le Corbusier (Figure 6). Il faut expliquer qu'il n'y a pas d'oeuvres relatives aux volumes 4 (1938-1946) et 8 (1965-1969).

Il s'agit d'une première sélection qui devra être vérifiée point à point, de manière systématique et approfondie. Dans ce cas, quelques unes de ces oeuvres pourront être excluses et d'autres pourront être incluses à l'ensemble, selon le développement des recherches. De manière identique, l'ensemble de critères de sélection pourra être adapté. Étant donné que l'oeuvre de Le Corbusier est vaste, large et variée, il sera éventuellement nécessaire limiter l'analyse des références projectuelles, spécialement dans cet article, qui s'agit d'une recherche en développement, et ayant la considèration d'atteindre le plus grand nombre de critères de relation, listés ci$\operatorname{dessus}^{26}$.

Pour cet article, on a réalisé un exercice schématique pour vérifier quelles oeuvres peuvent atteindre le plus grand nombre de critères de relation entre le projet de l'ambassade, ce qui a resulté en une série d'autres panneaux (Figures 5 à 18). Un dernier panneau synthétise et se superpose toutes les analyses, pour vérifier lesquelles de ces oeuvres atteignent le plus grand nombre de critères de relation avec le projet de l'ambassade.

L'Ambassade de la France à Brasilia est la première et la seule ambassade projeté par Le Corbusier. Les dessins sont datés de 1964 à 1965, c'est-à-dire, ils ont été reálisés deux ans après le retour de son dernier voyage au Brésil en 1962, quand il aurait été « commandé ». Comme déjà mentionné, en 1961 l'architecte j’avait déjà été contacté par le gouvernment suisse pour projeter et construire son Ambassade à Brasilia; cependant, le projet n'a pas été développé par Le Corbusier, qui l'aurait refusé, probablement parce qu'il avait beaucoup d'autres travaux en cours à l'époque. Il n'y a donc pas un terme de comparaison, relatif à un programme, entre le projet pour l'Ambassade de la France au Brésil et les autres travaux de Le Corbusier.

Les dessin (plantes, coupures et élévations) et les photos de la maquette du projet de l'Ambassade de France montrent que le programme a été organisé en deux volumes de façon clairement différente: un prisme cubique avc quatre étages, destiné à la résidence de l'Ambassadeur; et un volume cylindrique avec sept étages, destiné à la Chancellerie. Les volumes sont indépendents et, d'une certaine manière, antagoniques: il compose, à la fois, une synthèse plastique-visuelle qui attendent, en séparé et très bien, les complexent questions du projet et des questions fonctionnelles du programme.

La plante circulaire pleine est une nouveauté dans l'oeuvre de Le Corbusier. Les plantes partiellemnt circulaires sont présentes en aspects, ou secteurs, des oeuvres antérieures de Le Corbusier: dans ses premiers projets, comme la Villa Schwob (1916) à Chaux-de-Fonds en Suisse, et dans la Cité du Refuge (1932) à Paris; et elles

\footnotetext{
${ }^{26}$ Dans des possibilités, les oeuvres sélectionnées seront visitées et photographiées, et dans chaque cas la bibliographie disponible et les recherches en dpeveloppement sur ces oeuvres seront vérifiées.
} 
sont de nouveau présentes dans ses dernières oeuvres, comme dans le Palais de L'Assemblée (1955) à Chandigarh en Inde. Un cas spécial est l'église Saint Pierre (1960) à Firminy-Vert en France où la tour cylindrique-conique de section circulaire est née de la transformation d'une plante carrée.

La connexion entre les deux volumes de l'Ambassade se produit à travers le plan rectangulaire. L'implantation définit les routes internes à travers un dessin sinueux et organique, qui traverse en diagonale le lot d'une extrémité à l'autre. Le Corbusier avait déjà conçu des routes et des rues internes et des rampes avec des formes sinueuses, reliant des volumes, dans ses œvres ultérieures, telles que: dans le Centre de Calculs Électroniques Olivetti (1963) à Rho-Milan, Italie; dans l'Unité d'Habitation et dans l'Église Saint Pierre (1960), tous les deux à Firminy-Vert, et dans le Palais des Congrès (1964) à Strasbourg, en France.

Des poches de parking sectorisées sont situées à côté de chaque volume; tout près de la Chancellerie il y a une deuxième poche afin de répondre à la hausse de la demande pour les véhicules des employés et des visiteurs de l'immeuble impliqués dans les activités administratives et les activités protocolaires.

Le volume cubique de la résidence est situé à l'arrière du terrain, profitant de la vue contemplative du paysage naturel du lac qui borde le bout du terrain; la maison et la piscine ont une position plus réservée et privée, et la piscine ne pourrait pas être vue par quelqu'un qui s'approchait du lieu. Le volume cylindrique de la chancellerie se trouve en face du terrain, à côté de l'accès et du contexte urbain.

Le volume prismatique de la résidence a ses plus grandes faces positionnées parallèlement à la face inférieure de la ligne de fond du terrain rectangulaire. Les deux plus grandes faces sont composées de panneaux de verre protégées par brises soleil de béton, tandis que les deux faces plus petites sont des pignons aveugles de béton armé. A l'intérieur du bâtiment il est possible d'envisager la vue sur la ville planifiée par Costa et Niemeyer, deux disciples de Le Corbusier.

Le volume cylindrique de la chancellerie est presque complètment entouré par une peau en verre protegée du soleil fort de la région par des brises soleil en béton donnant sur presque tous les alentours. Seule la zone pour les escaliers, les salles de bains et les ascenseurs forment un tour fermé en béton armé placé à l'avant du champ, c'est-à-dire, sur le côté sud du terrain, en face de la ville.

Dans les deux volumes les brises soleil indiquent une structure indépendante et déconnectée du volume, représentant une deuxième peau qui entoure chaquer bâtiment à côté de lui.

L'utilisation de la façade indépendante du reste du volume édifié et composé par des brises soleil a été d'abord utilisé par l'architecte dans la Maison du Docteur Curutchet (1949) à La Plata, en Argentine. Et les brises soleils sur les façades indépendantes ou non apparaisent dans des œuvres antérieures au projet de l'Ambassade, et qui sont liées à l'architecte comme, par exemple, le Ministère de l'Éducation et de la Santé (1936-1945) à Rio de Janeiro, au Brésil; dans la Villa Shodhan et le Palais des Filateurs (1951), les deux situés à Ahmedabad, en Inde; dans le Palais d'Assemblée (1955) à Chandigarh, en Inde; dans la Maison du Brésil (1957) à Paris, en France; dans le Visual Arts Center à l'Université de Harvard (1961) à Cambridge, aux États-Unis; dans le Centre des Calculs Électroniques Olivetti (1963) à Rho-Milan, en Italie, et dans le Palais des Congrès (1964) à Strasbourg, en France.

Une autre charactéristique commune aux deux volumes c'est l'utilisation de la structure dom-ino, équivalente au prototype de la Maison Dom-ino présenté par l'architecte pour la première fois en 1914. Cette même structure est également répandue dans d'autres travaux liés à l'architecte comme, par exemple, la Ville Savoye (1928) à Poissy, France; dans la Villa Baizeau (1928) à Carthage, en Tunisie; dans la Cité du Refuge (1932) à Paris, France; dans le Ministère de l'Éducation et de la Santé (1936-1945) à Rio de Janeiro, au Brésil; dans la Maison 
du Docteur Curutchet (1949) à La Plata, Argentine; dans la Villa Shodhan et le Palais des Filateurs (1951), à Ahmedabad, en Inde; dans le Palais d'Assemblée (1955) à Chandigarh, Inde; dans la Maison du Brésil (1957) à Paris, France; dans l'Église Saint Pierre (1960) à Firminy-Vert, France; dans le Visual Arts Center de l'Université de Harvard (1961) à Cambridge, aux États-Unis, dans le Centre des Calculs Électroniques Olivetti (1963) à Rho-Milan, en Italie, et aussi dans le Palais des Congrès (1964) à Strasbourg, en France.

Avec l'utilisation de la structure dom-ino, Le Corbusier peut concevoir la dalle de chaque étage librement, de sorte que parfois cela progresse et implique les pilotis ou parfois elle recule et crée des coupures qui génèrent des étages avec des pieds-droits doubles ou triples et des cours découverts dans le dernier étage. Par conséquent, l'usage de la structure, le plan et les façades sont liberés, comme nous pouvons observer par la position des divisions internes et par les panneaux en verre qui scèllent les deux immeubles.

Le pied-droit double ou triple est aussi représenté dans des œuvres antérieures liées à l'architecte, telles que le Ministère de l'Éducation et Santé (1936-1945) à Rio de Janeiro, Brésil; la Maison du Docteur Curutchet (1949) à La Plata, Argentine; Villa Shodhan et Palais des Filateurs (1951) à Ahmedabad, en Inde; le Palais d'Assemblée (1955) à Chandigarh, en Inde; l'Église Saint Pierre (1960) à Firminy-Vert, France; dans le Visual Arts Center de l'Université de Harvard (1961) à Cambridge, aux États-Unis, et le Palais des Congrès (1964) à Strasbourg, France.

Les cours intérieures avaient déjà apparus: dans la Villa Savoye (1928) à Poissy, France; dans la Villa Baizeau (1928) à Carthage, Tunisie et aussi dans un de ses derniers projets, l'Hôpital (1964) à Venise, Italie.

Un autre élément impotant du projet ce sont les deux rampes d'accès à la résidence de l'Ambassadeur. Chaque rampe est située perpendiculairement à chacune des plus grandes faces du prisme et sur des côtés opposés. Ces rampes rappellent celles projectées par Niemeyer pour le Congrès National à Brasilia. Les rampes projectées par l'architecte brésilien sont représentées par une feuille relativement mince de béton armé, desserrée du sol, et similaire à un tapis flotant. Par contre, les rampes de l'architecte suisse-français sont denses et lourdes, clouées au sol. Cette distinction entre le même élément projecté de manières différentes par les deux architectes fait que la phrase dite par Le Corbusier à Italo Campofiorito à l'occasion de sa visite à Brasilia - et qui fait référence à la délicatesse des formes des œuvres réalisées par les architectes brésiliens en comparaison avec les siennes, plus grossières - soit plus évidente et elle nous permet la réflexion que, à la fin de sa carrière, Le Corbusier serait probablement en train d'assimiler une part de la contribuition de ses disciples, même les brésiliens.

Les rampes intérieures qui composent une promenade architecturale et les rampes extérieures d'accès au volume avaient déjá été utilisées par l'architecte suisse-français dans ses œuvres antérieures comme, par exemple, dans la Villa Savoye (1928) à Poissy, France; dans la Villa Baizeau (1928) à Carthage, en Tunisie; dans la Maison du Docteur Curutchet (1949) à La Plata, Argentine; dans la Villa Shodhan et le Palais des Filateurs (1951) à Ahmedabad, en Inde; dans le Palais d'Assemblée (1955) à Chandigarh, Inde; dans l'Église Saint Pierre (1960) à Firminy-Vert, France; dans le Visual Arts Centre de l'Université de Harvard (1961) à Cambridge, aux États-Unis; dans le Centre des Calculs Électroniques Olivetti (1963) à Rho-Milan, en Italie et dans le Palais des Congrès (1964) à Strasbourg, en France.

Si on examine chaque volume de plus près on voit que, dans le cas du prisme, les séquences, c'est-à-dire, les axes des pilotis suivent toujours avec la même quantité: trois dans le sense longitudinal et quatre dans le sense transversal; toutefois, ce n'est pas le cas du cylindre. Dans le deuxième, il est possible de noter la variation dans les deux directions. Dans le sense longitudinal il y a une séquence, c'est-à-dire, un axe avec deux pilotis, et les suivants avec sept, cinq, quatre et trois, successivement. Dans la direction transversale (de gauche à droite): un, un, un, trois, quatre, quatre, quatre, un, un et un. C'est correcte en raison de la forme bidimensionelle du cercle 
qui ne permet pas la même quantité de pilotis au même rythme entre eux et c'est nécessaire diminuer au fur et à mesure qu'on se distancie de son centre.

Cette variation résulte aussi de l'emsemble composé par l'escalier, les ascenseurs et les salles de bains qui composent une structure en forme de «boîte » fermée qui fonctionne comme un grand pilier qui collabore, tout avec les pilotis, pour soutenir l'emsemble de la construction.

On peut noter aussi que Le Corbusier n'a pas suivi la même rigueur de rythme entre les pilotis du cylindre avec lequel il avait conçu le prisme. Pour le cylindre, Le Corbusier propose des rythmes différents donnant plus de liberté à celui-ci qu'au prisme et c'est pour cette raison qu'on aperçoit quatre pilotis « libérés » qui interrompent le rythme et traversent la façade indépendente des brises soleil. Cette variation ne serait pas perceptible pour l'utilisateur qui se trouve à l'intérieur de l'immeuble, au cas celui-ci aurait été construit. Cette variation peut seulement être notée par l'observateur de ses plans.

Selon la déclaration de l'architecte français OUBRERIE $(2007)^{27}$, les derniers projets de Le Corbusier marquent la transition vers une architecture plus libre et inventive et, paradoxalement, plus structurée.

Pour cet auteur, un exemple significatif de cette nouvelle phase serait le projet de l'Ambassade de France, qui présenterait une dichotomie entre la rigueur de la Résidence de l'Ambassadeur et la liberté et l'invention de la Tour de la Chancellerie.

«Les projets qui suivent - comme l'illustre le projet de l'Ambassade de Brasilia, qui montre une dichotomie entre la rigueur exprimée dans la Résidence de l'Ambassadeur et la liberté et l'invention de la Tour de la Chancellerie - sont déjà des projets de transition vers une architecture à la fois plus libre et inventive et paradoxalement plus structurée. » (2007: 51)

\section{Conclusions}

Ce travail présente des conclusions partielles d'une étude en cours ayant pour objectif les relations professionnelles entre Le Corbusier et le Brésil qui ont été collectées, vérifiées et systématisées et le propos de cette étude est le projet non accompli de Le Corbusier pour l'Ambassade de France au Brésil (1965-65), en lui considérant sous la lumière de toute l'œuvre corbusiènne, et plus spécifiquement de ses œuvres des années 1960. La comparaison entre l'œuvre de l'Ambassade du Brésil et les autres œuvres corbusiènnes a visé l'investigation et une compréhension plus ample des choix et des chemins cristallisés par la créativité et l’inventivité de cet architecte si expérimenté.

Bien que les analyses précédentes n'aient pas encore permis de conclure que ses dernières années de vie, quand Le Corbusier travaillait sur le projet pour l'Ambassade de France, démontrent une nouvelle phase de sa trajectoire créative, on peut cependant suggérer, en considérant cet œuvre à la lumière de toutes les autres antérieures, qu'il soit possible de la comprendre comme um moment de «synthèse », dans lequel l'architecte revisite et recombine les thèmes, les formes, les matériaux et les stratégies présentes dans les projets élaborés dans toutes ses phases précédentes (puriste, de cinq points, de matériaux naturels des années 1930, du

${ }^{27}$ OUBRERIE, Jose. Notes sur la contribution créative de Guillermo Jullian à l'atelier Le Corbusier. In : Massilia. Annuaire des Études Corbusiennes. ARQ Ediciones / PUC-Chile : Santiago, Chile, 2007, p. 50-53. 
brutalisme...). et, si au début de sa carrière Le Corbusier assimile et transforme la constribution de ses maîtres, à la fin il sera certainement en train d'assimiler quelque chose de la contribution de ses disciples, y inclus les brésiliens, en travaillant sur un bouillon de culture dense et complexe.

\section{Pièces jointes}

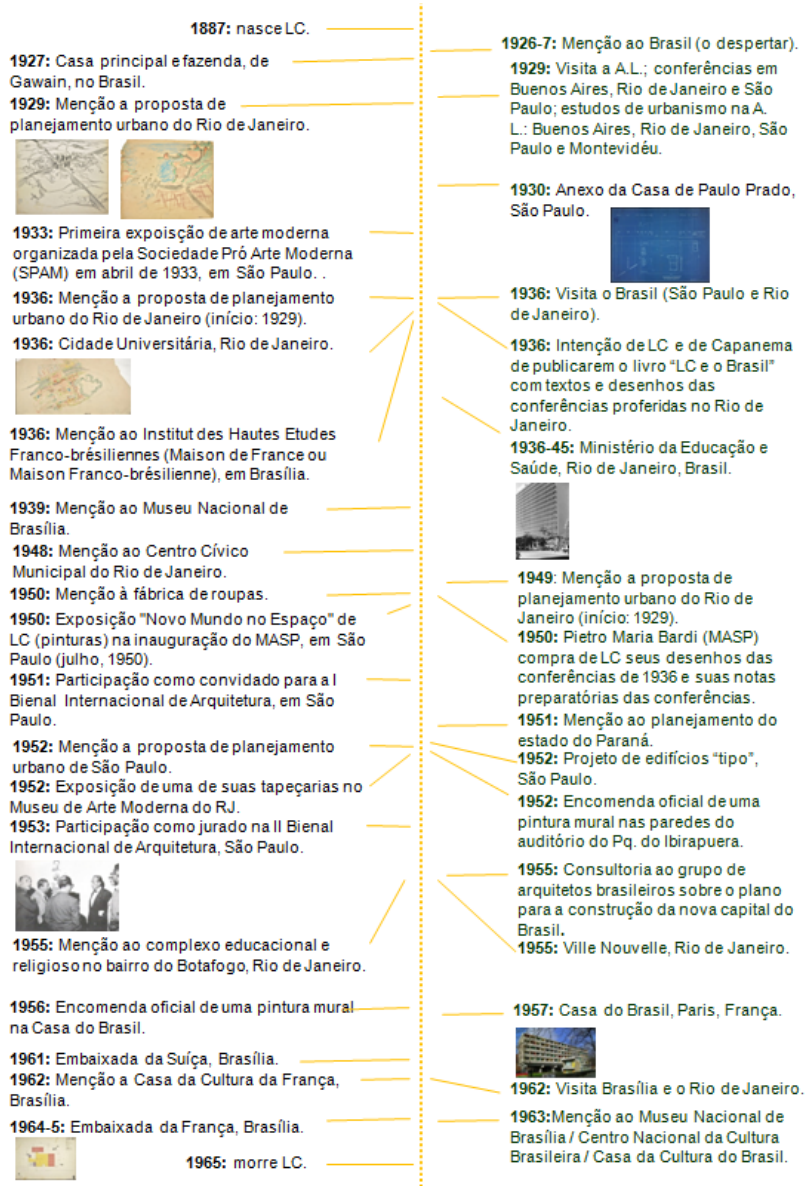

1. Chronologie. Source: Préparé par CHIARELLI basé sur les informations fournies dans les sources consultées par mai/2015. 
“São Paulo, mai 8, 1950.

Le Corbusier

35 rue de Sèvres

PARIS - Vlme.

Cher M. Le Corbusier :

Suite à votre lettre du 28 avril j'ai a vous communiquer le suivant concernant les deux idées posées.

$1^{o}$ - La question de la 'casa franco-bresilienne"’ à Rio il y a longtemps on n'en parle plus. Je ne sais pas bien si la question est completemente tombée ou à quel point elle se trouve, en tout cas je m'informerais à propos et j'aurai plaisir à vous écrire.

$2^{\circ}$ - Concernant les grands dessins éxecutés pendant vos conferences à Rio en 1936 je suis vraiment très interessé pour savoir à quel prix vous pourriez les céder au Musée. Cela nous interesse beaucoup et je crois bien qui îls sont la clef même de l'architecture moderne brésilienne. Comme documentation et démonstration ces dessins nous seraint très utiles. Nous attendons votre proposition pour vous donner une réponse définitive.

$3^{\circ}$ - Votre exposition de Boston doit embarquer dans ces jours pour Santos. La salle dont elle sera exposé est prête. Je vous envoyerrai des photos quand elle sera monté pourque vous poussiez juger de la façon elle sera presenté. L'Instituto de Arte Moderna de Buenos Aires ne peut pas presenté l'exposition. Nous tacherons de contacté avec d'autres Musées.

A vous lire bientôt je vous pris de bien vouloir agréer mes salutation très amicales.

P. M. Bardi-directeur"

2. La lecture du contenu de la lettre de Pietro Maria Bardi pour Le Corbusier, 08/05/1950. Source: Bibliothèque et Centre de Documentation du MASP. 
“May 10, 1950

Mr. P. M. Bardi

Museu de Arte

Rua 7 de Abril No 216

São Paulo, Brasil

Dear Sir:

In reply to your kind letter of March 29th, we are glad to let you know that it will be agreeable to us to receive payment in French Francs for the paiting by Le Corbusier, 'Nature morte pleine d'espace', wich you wish to acquire from us.

The price of the picture is, as you know, one thousand dollars, and we would be pleased to received the equivalent in Francs.

We assume that you will take care of the cost of transportation, and we hope to receive soon pour shipping instructions.

Looking forward to hearing from you, we remain.

Sincerely yours,

A. P. ROSENBERG \& CO., INC.

Florence Walters"

3. La lecture du contenu de la lettre de Florence Walters pour Pietro Maria Bardi, 10/05/1950. Source: Bibliothèque et Centre de Documentation du MASP. 
"May 18, 1950

Mr. P. M. Bardi, Director

Museu de Arte

Rua 7 de Abril, 216

São Paulo, Brazil

Dear Mr. Bardi:

Your letter of May 10 enclosing your check in the amount of $\$ 1070$ arrived on May 17. The six cases comprising the Le Corbusier exhibition were delivered to the Moore-McCormack Lines today. The ship on which the exhibition will be coming to Santos is the "Moore-Mac-Surf"'; it is due to sail on Monday, May 22 from Boston, an unexplained delay of tree days.

Enclosed you will find a list of the cases and the material contained there in. You will note that be of help to you. In any case, on the back of every photographic panel, and every panel of text, is a diagram which shows the order of installation. I am including a description of our installation here.

Finally, enclosed here with is a copy of a letter from our Customs Brokers in regard to the necessity of your making a declaration before the American Consul on Form 204 in order to bring the material back to this country duty free.

May I take this opportunity to tell you how delight we are to send the Le Corbusier exhibition to São Paulo. We wish you every success for your showing at the Museu de Arte.

Most sincerely,

Natalie Marston

PS : The cases are marked as follows: M.A.S.P, Santos. We have also affixed labels bearing tha name and address of the Museu de Arte."

4. La lecture du contenu de la lettre de Natalie Marston pour Pietro Maria Bardi, 18/05/1950. Source: Bibliothèque et Centre de Documetation du MASP. 


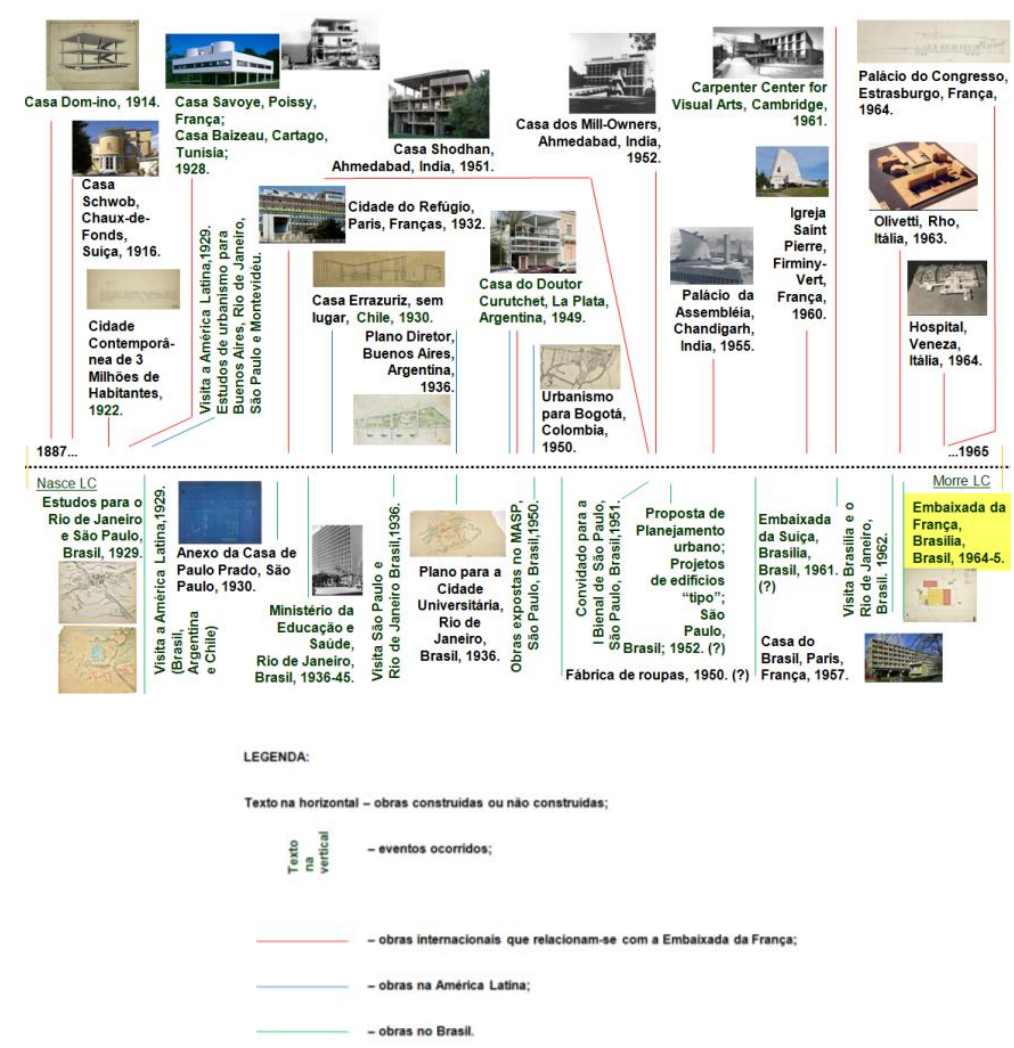

5. Travaux et événements. Source: Préparé par CHIARELLI basé sur les informations fournies dans les sources consultées par mai/2015.

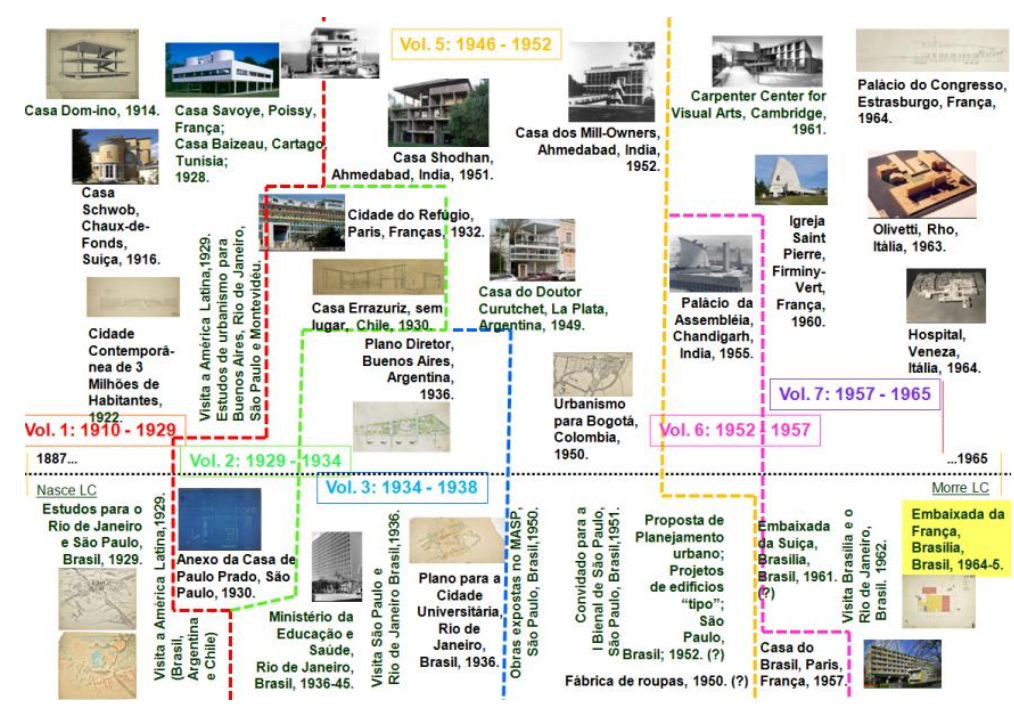

6. Travaux et événements divisés par les volumes . Source: Préparé par CHIARELLI basé sur les informations fournies dans les sources consultées par mai/2015. 


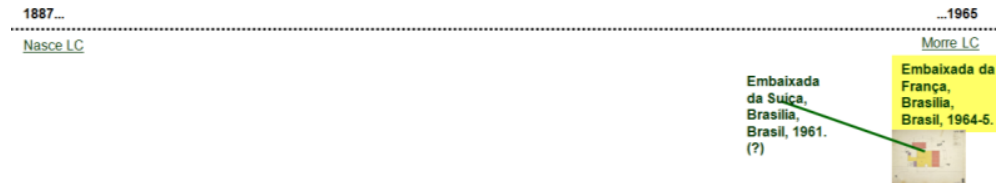

7. Ambassades projets. Source: Préparé par CHIARELLI basé sur les informations fournies dans les sources consultées par mai/2015.

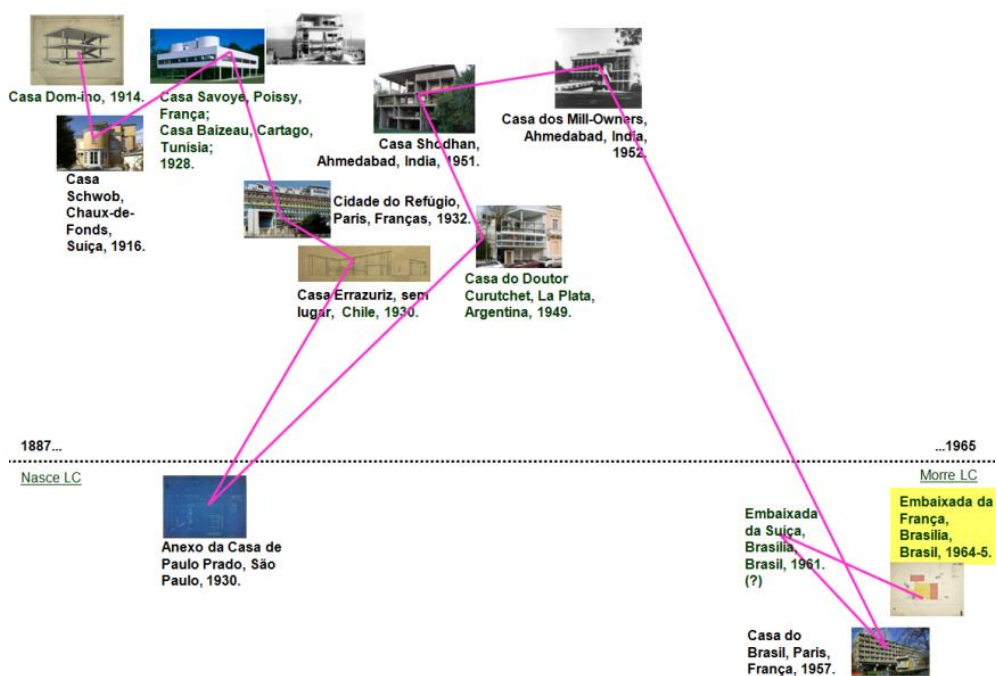

8. Programme résidentiel. Source: Préparé par CHIARELLI basé sur les informations fournies dans les sources consultées par mai/2015.

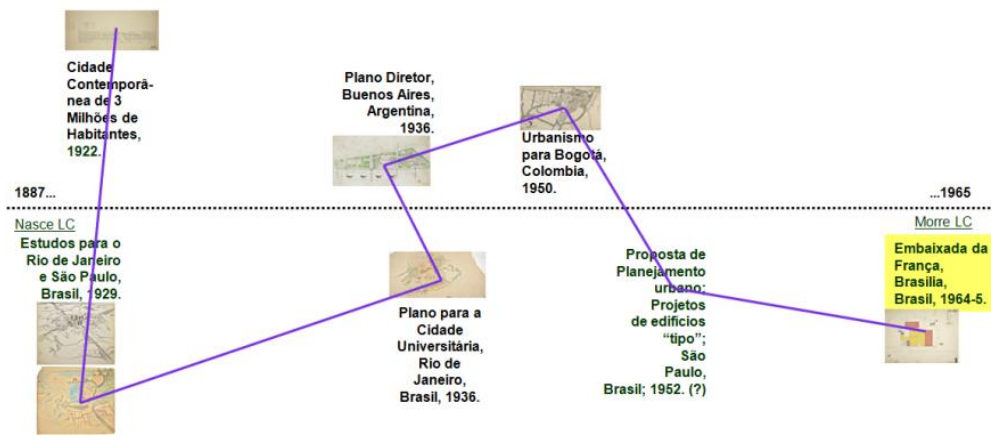

9. Relation à l'échelle humaine. Source: Préparé par CHIARELLI basé sur les informations fournies dans les sources consultées par mai/2015. 


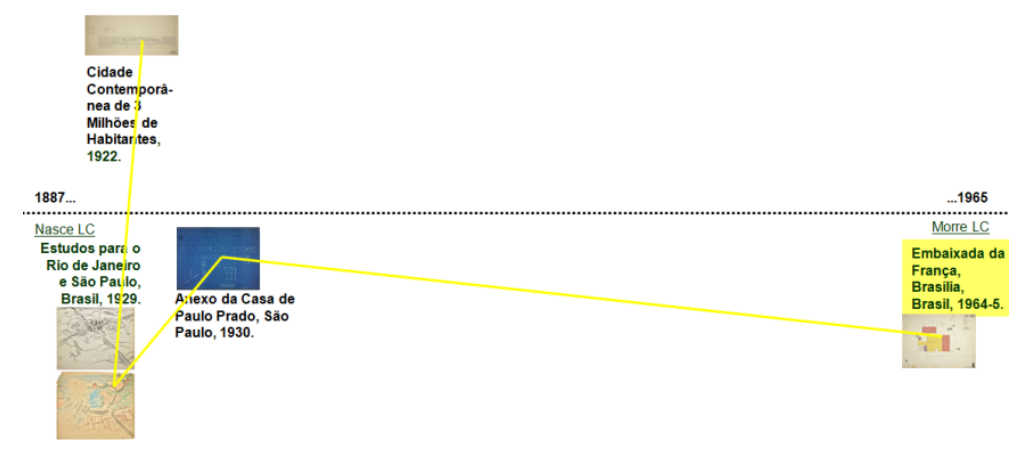

10. Premières relations avec le Brésil. Source: Préparé par CHIARELLI basé sur les informations fournies dans les sources consultées par mai/2015.

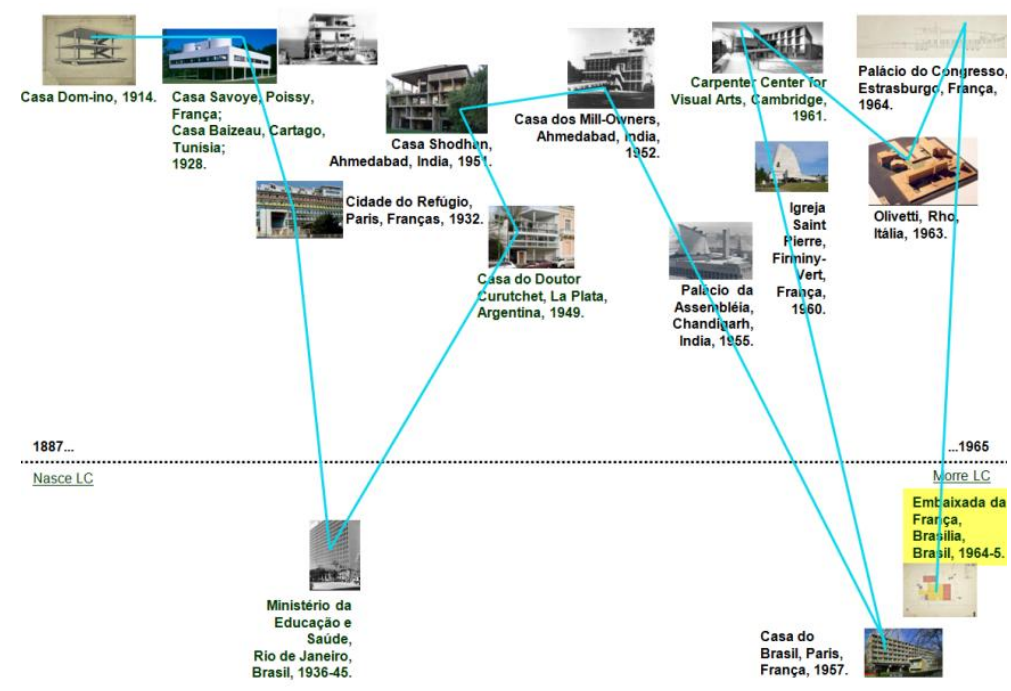

11. La estructure dom-ino. Source: Préparé par CHIARELLI basé sur les informations fournies dans les sources consultées par mai/2015.

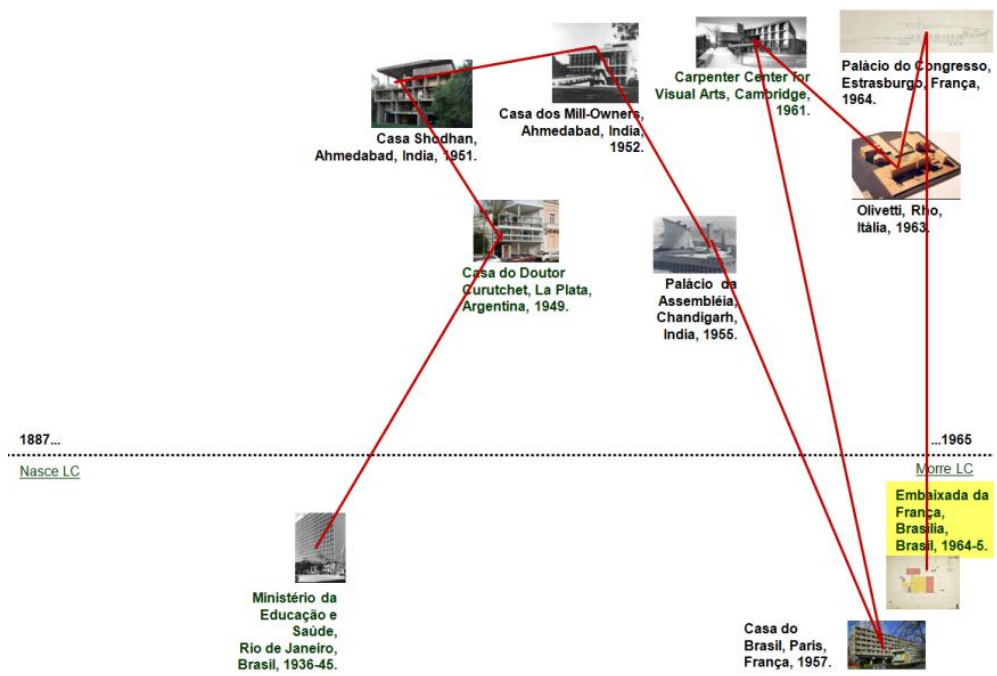

12. Présence des brises de soleil. Source: Préparé par CHIARELLI basé sur les informations fournies dans les sources consultées par mai/2015. 


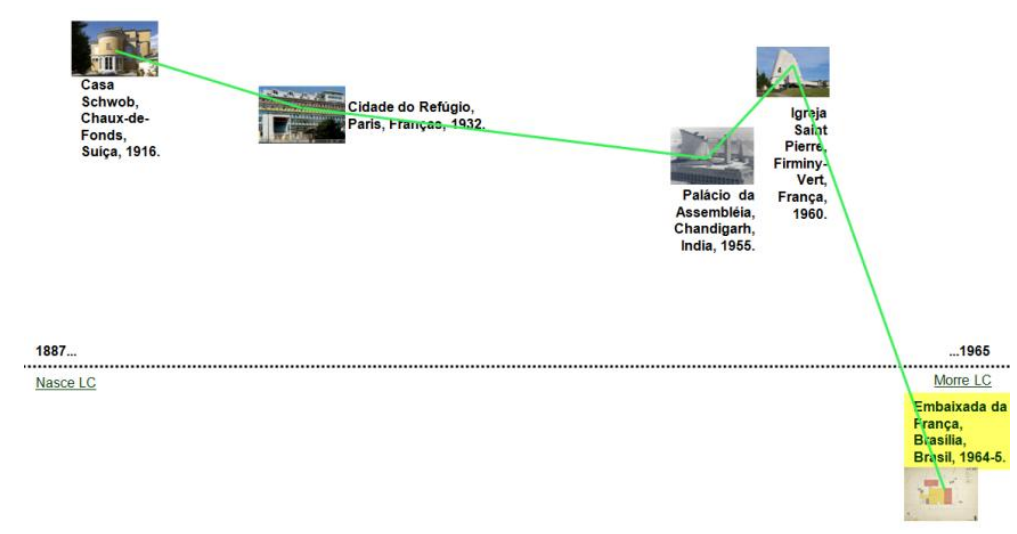

13. Présence de volumes avec plante circulaire. Source: Préparé par CHIARELLI basé sur les informations fournies dans les sources consultées par mai/2015.

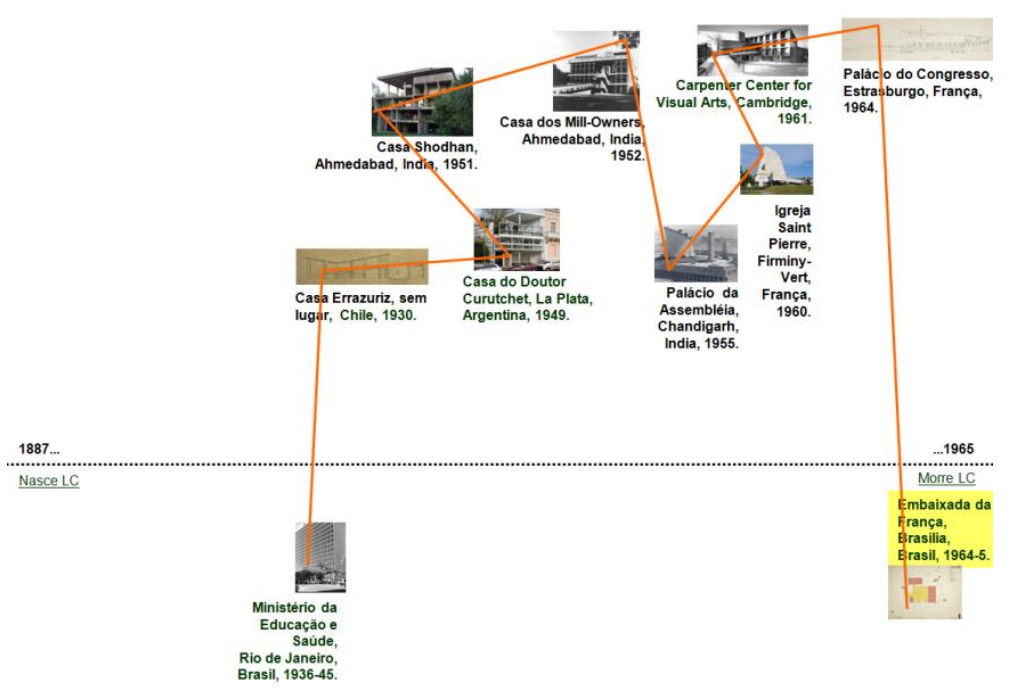

14. Présence de double hauteur ou triple. Source: Préparé par CHIARELLI basé sur les informations fournies dans les sources consultées par mai/2015.

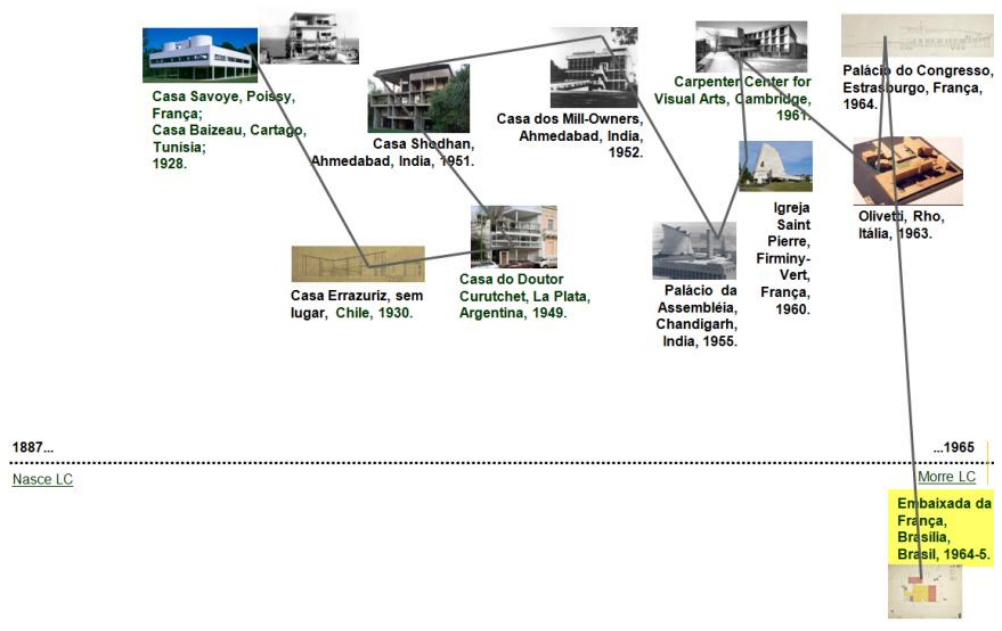

15. Rampes d'accès / Promenade architecturale. Source: Préparé par CHIARELLI basé sur les informations fournies dans les sources consultées par mai/2015. 


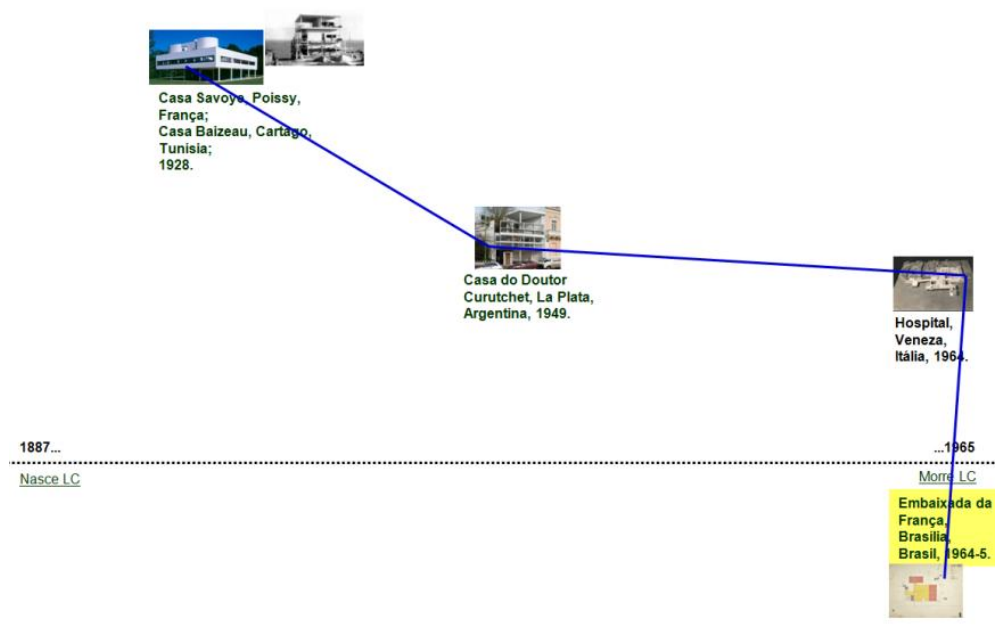

16. La présence de plaques perforées / patios dans l'interiur. Source: Préparé par CHIARELLI basé sur les informations fournies dans les sources consultées par mai/2015.

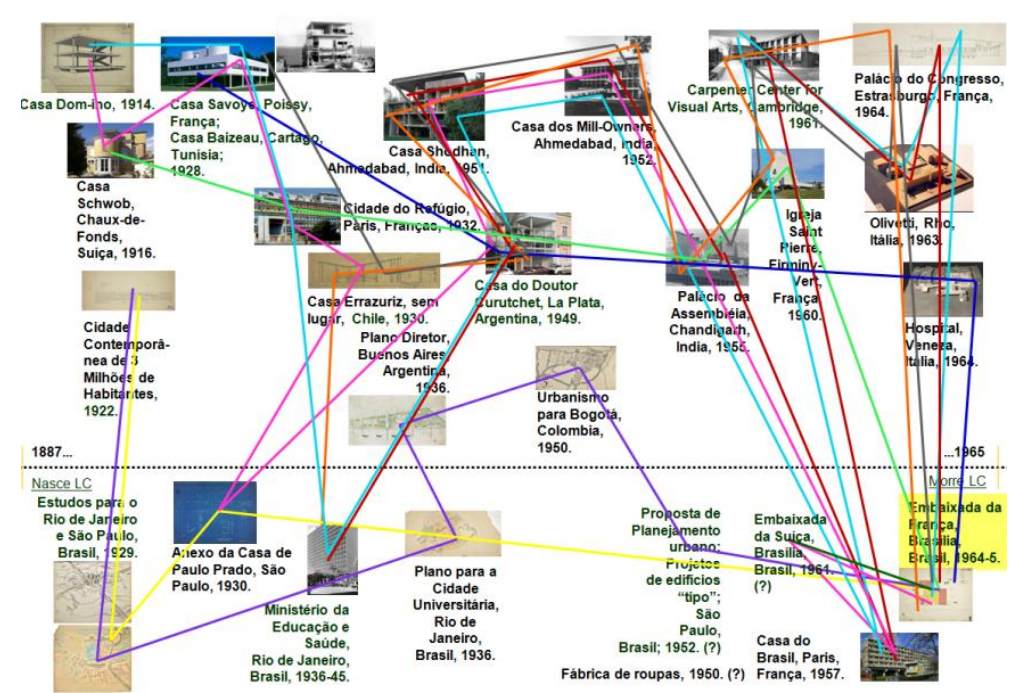

17. Résumé des analyses précédentes. Source: Préparé par CHIARELLI basé sur les informations fournies dans les sources consultées par mai/2015. 


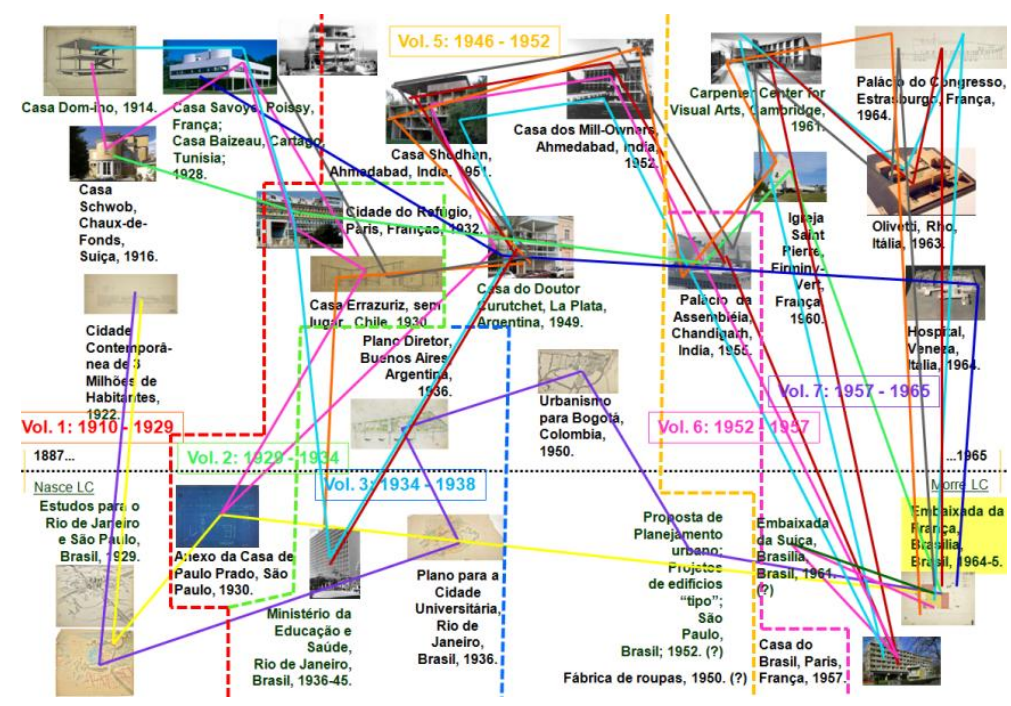

18. Résumé des analyses précédentes. Source: Préparé par CHIARELLI basé sur les informations fournies dans les sources consultées par mai/2015.

\section{Bibliographie / Références}

BARDI, Pietro Maria. Leitura crítica de Le Corbusier. Sao Paulo: Ed. Habitat, 1950.

CHATEAU, Francisco. Yellow Peripherical Distinction. Guillermo Jullian en el encuentro del Team X en Berlin, 1973. In: Massilia. Annuarie des L'Études Corbusiennes. ARQ Ediciones / PUC-Chile: Santiago, Chile, 2007, p. 76-89.

DI MATTEO, Colette; TIDORI, Jean-Martin (org.). Embaixada da França / L'Ambassade de France: Brasília. Sao Paulo: Imprensa Oficial do Estado de São Paulo, Instituto Totem Cultural, 2009.

GUERRERO, Ingrid Quintana. Filhos da Rue de Sèvres: relações e trocas dos colaboradores latino-americanos de Le Corbusier em Paris. Tese ( $\mathrm{PhD}$, em cours, dans l'Architecture et de 1'Urbanisme), Université de Sao Paulo, Sao Paulo.

JEANNERET-GRIS, Charles-Edouard. Le Corbusier: oeuvre complète 1910-1965. Zurique: Artemis, 1967. 8 volumes.

LE CORBUSIER. Precisões: sobre um estado presente da arquitetura e do urbanismo. Tradução: Carlos Eugênio Marcondes de Moura. Sao Paulo: Cosac Naify, 2004. [Ed. orig., Précisions: sur un état présent de l'architecture et de l'urbanisme, 1930].

OUBRERIE, Jose. Notes sur la contribution crétive de Guillermo Jullian à l'atelier Le Corbusier. In: Massilia. Annuarie des L'Études Corbusiennes. ARQ Ediciones / PUC-Chile: Santiago, Chile, 2007, p. 50-53.

OYARZÚN, Fernando Pérez. Le Corbusier y Sudamérica: viajes y proyectos. Massilia. Santiago do Chile: Ediciones ARQ, 1991.

PUPPI, Marcelo. Espaços inacabados: Le Corbusier, Lúcio Costa e a saga da Casa do Brasil, 1953-1956. Magazine ARQTEXTO (PROPAR/UFRGS), Porto Alegre, No 12, 2008, p.160 - 203.

SANTOS, Cecília Rodrigues dos; PEREIRA, Margareth Campos da Silva; PEREIRA, Romão Veriano da Silva; SILVA, Vasco Caldeira. Le Corbusier e o Brasil. Sao Paulo: Tessela, 1987.

SEGAWA, Hugo. Entrevistas e depoimentos: A viagem de Le Corbusier ao Brasil, em 1962. In: Projeto, Sao Paulo, n. 102, 1987.

Collection du Centre de Documentation et Bibliothèque du Musée d'Art de Sao Paulo - MASP

Collection de la Fondation Le Corbusier

Adresse virtuelle: www.fondationlecorbusier.fr 